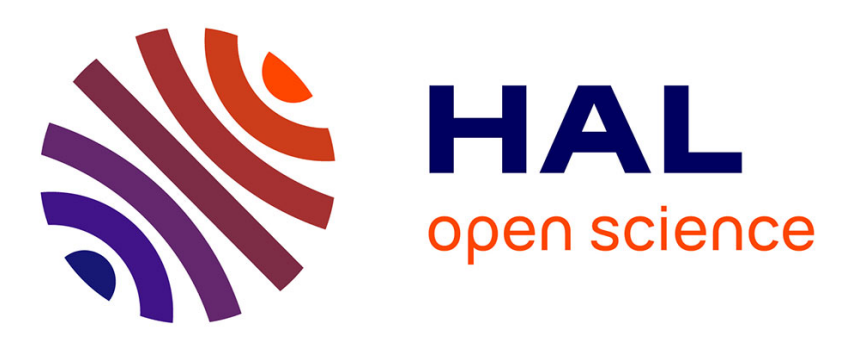

\title{
MODIFICATIONS APPORTÉES A LA MÉTHODE DE DIAGNOSTIC DU LAIT DE VACHE DANS LE LAIT DE FEMME \\ P. Romeyer
}

\section{- To cite this version:}

P. Romeyer. MODIFICATIONS APPORTÉES A LA MÉTHODE DE DIAGNOSTIC DU LAIT DE VACHE DANS LE LAIT DE FEMME. Le Lait, 1950, 30 (295_296), pp.249-252. hal-00928015

\section{HAL Id: hal-00928015 https://hal.science/hal-00928015}

Submitted on 1 Jan 1950

HAL is a multi-disciplinary open access archive for the deposit and dissemination of scientific research documents, whether they are published or not. The documents may come from teaching and research institutions in France or abroad, or from public or private research centers.
L'archive ouverte pluridisciplinaire HAL, est destinée au dépôt et à la diffusion de documents scientifiques de niveau recherche, publiés ou non, émanant des établissements d'enseignement et de recherche français ou étrangers, des laboratoires publics ou privés. 


\title{
MODIFICATIONS APPORTÉES A LA MÉTHODE DE DIAGNOSTIC DU LAIT DE VACHE DANS LE LAIT DE FEMME
}

\author{
par
}

\section{P. ROMEYER}

Chef de laboratoire du lait et du Lactarium de St-Etienne

La méthode de diagnostic du lait de vache dans le lait de femme, déjà communiquée (Le Lait, 1949, 29, 576) et pratiquée très souvent au Laboratoire, a révélé, à l'usage, quelques imperfections. Je me suis done efforcé d'en améliorer la technique, en la rendant plus simple et plus rapide à la fois, tout en éliminant de mon mieux les causes d'erreurs dues à l'interprétation.

Voici done l'exposé de la méthode modifiée.

\section{Pratique de l'opération}

1 er Temps. - Floculation (technique de FouILHOUZE).

On prend $2 \mathrm{~cm}^{3}$ de lait suspect qu'on coagule dans un bécher de $100 \mathrm{~cm}^{3}$ à l'aide de 2 réactifs :

1 solution de ferrocyanure de potassium à $15 \% \ldots \ldots \ldots \ldots \ldots 0 \mathrm{~cm}^{3} 2$

1 solution d'acétate de zine à $11 \% \quad \ldots \ldots \ldots \ldots \ldots \ldots \ldots, 0 \ldots \mathrm{em}^{3} 4$

On agite, la floculation se produit. On dilue le floculat par addition de $25 \mathrm{~cm}^{3}$ d'eau distillée environ. On filtre, le liquide s'écoule limpide. Le filltrat est reęu dans une fiole jaugée de $100 \mathrm{~cm}^{3}$. L'opération terminée, on complète à $100 \mathrm{~cm}^{3}$ avec de l'eau distillée.

$2^{\mathrm{e}}$ Temps. - Formation du complexe phospho-molybdique.

A $0 \mathrm{~cm}^{3} 5$ d'une solution de molybdate d'ammoniaque dans l'acide sulfurique de composition :

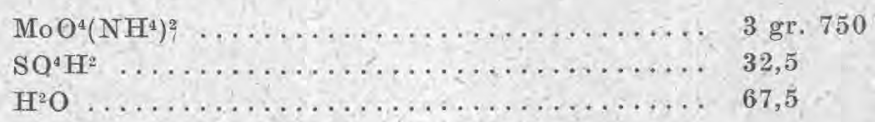

On ajoute $5 \mathrm{~cm}^{3}$ du filtrat précédent. On chauffe à $75^{\circ}$ quelques secondes. Dans ces conditions, les phosphates minéraux se combinent au molybdate d'ammoniaque en donnant un précipité de phospho-molybdate d'ammoniaque qui teinte plus ou moins la solution en jaune, suivant que le titre en phosphate est plus ou moins élevé.

3e Temps. - Réduction du complexe molybdique.

Alors que le molybdate d'ammoniaque n'est pas réduit par le chlorure stanneux en milieu chlorhydrique, dans les conditions de l'expérience, le complexe phospho-molybdique est réductible par ce réactif de composition : 
Chlorure stanneux pur $\ldots \ldots \ldots \ldots \ldots \ldots \ldots \ldots$ gr. 200

Acide chlorhydrique pur .............. $2 \mathrm{~cm}^{3}$

Eau distillée ..................... $10 \mathrm{em}^{3}$

On ajoute donc 2 gouttes de solution stanneuse au filtrat précédent. Apparaît alors une coloration bleue d'autant plus intense que la teneur en phosphore est plus élevée.

\section{$4^{\mathrm{e}}$ Temps. - Les échantillons témoins.}

Comme dans le procédé initial, il suffit d'établir une gamme étalon de différents échantillons purs et fraudés pour pouvoir juger s'il y a eu addition de lait de vache au lait de femme et dans quelles proportions?

Pour la confection de cette gamme de référence, je propose de faire la réaction avec plusieurs laits de femme purs afin de vérifier la constance du taux en phosphore minéral du lait de femme. Une fois ces témoins établis, frauder artificiellement un échantillon de lait de femme pur par des quantités progressives de lait de vache, de chèvre ou de brebis déterminant ainsi les poucentages de lait fraudé :

$$
5-10-20-30 \% \ldots \ldots \ldots \ldots \ldots \ldots \ldots \ldots \ldots \ldots, 80-90 \%
$$

A cette série de tubes, il conviendrait d'ajouter un tube témoin, ce tube servant à vérifier la pureté des différents réactifs, en particulier de l'eau de dilution. En ce cas le témoin se compose de :

$$
\begin{aligned}
& \text { Eau distillée ....................... } 5 \mathrm{em}^{3}
\end{aligned}
$$

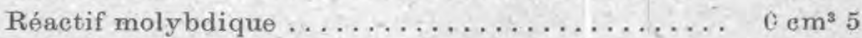

$$
\begin{aligned}
& \text { Solution de chlorure stanneux } \ldots \ldots \ldots \ldots \ldots \ldots .2 \text { gouttes }
\end{aligned}
$$

Après addition du chlorure d'étain, on ne doit percevoir aucune coloration.

5e Temps. - Interprétation des résultats.

Les opérations terminées, il est facile, partant d'un échantillon de lait suspect, de déterminer visuellement s'il y a fraude ou non.

Point n'est besoin d'utiliser de colorimètre ; pour un œil exercé il est aisé de situer, après comparaison de l'échantillon suspect, à chacun des témoins précédemment obtenus, sa place dans la gamme d'échantillons colorés. Lorsqu'on a atteint une égalité des colorations, on peut dire que l'échantillon suspect présente une fraude équivalente à celle du témoin.

\section{Notes générales sur l'utilisation du procédé}

1. La verrerie utilisée doit être préalablement rincée à l'eau 
distillée pour éviter les causes d'erreurs dues à la présence de phos" phates dans l'eau d'alimentation de ville.

2. La stabilité des échantillons témoins n'est réalisable qu'à condition, de les placer à une température inférieure à $5^{\circ}$, à l'obscurité et à l'abri de l'air. La pratíque a montré qu'il est difficile de créer des témoins artificiels bleus soit à l'aide d'ammoniaque et de sulfate de cuivre, soit de bleu de bromothymol ou autres colorants, toutaussi labiles et instables que les modèles originaux. Ces derniers pourront d'ailleurs être reproduits de temps en temps assez facilement.

3. La solution de chlorure stanneux voit avec le temps son pouvoir réducteur décroître, même en plaçant le réactif à l'abri de l'air et de la lumière. Il est done prudent de le reñouveler une fois par semaine environ. Cependant la pratique montre qu'en utilisant le sel (chlorure stanneux) à l'état pur, on peut utiliser le même réactif pendant un mois.

4. Quelques mots enfin sur la précision de la méthode et sur son utilisation. Cette méthode permet de détecter $5 \%$ de lait de vache additionné au lait de femme, précision qui semble suffisante car une fraude inférieure au taux de $5 \%$ et même de $10 \%$ ne serait d'aucun profit pour la personne qui s'en rendrait coupable. Les essais ont porté sur des laits normaux. Pour les laits colostraux de teneur plus faible en phosphate, le procédé reste valable.

Quant à l'utilisation de la méthode, elle se généralise à tous les laits courants : vache, chèvre, brebis, tous ces laits peuvent être détectés facilement, leur teneur en phosphore minéral allant en croissant suivant l'énumération.

En outre, elle s'applique aux laits ayant subi un traitement thermique : laits de pasteurisation basse, haute, tyndallisés, ehauffés à $100^{\circ}$. Avantage primordial sur les méthodes précédemment en usage (séro-précipitation).

L'utilisation de cette méthode en vue de mettre en évidence un mouillage éventuel s'est avérée inopérante : la raison en est simple, le mouillage s'effectue avec une eau plus ou moins potable dont la teneur en phosphate n'est pas négligeable mais variable d'une eau à l'autre; d'autre part, il est peu probable que la personne qui mouillera le lait l'additionne d'eau distillée (exempte de sels minéraux). En ce eas, la méthode est à condamner en ce qui concerne le dépistage des laits mouillés.

Enfin, elle présente d'autres avantages' sensibles, elle est courte, l'ensemble des opérations ne dépasse pas 15 minutes, à l'opposé de la lenteur de la méthode aux sérums spécifiques.

Elle est simple. L'appareillage et les réactifs sont faciles à se 
procurer et peuvent être mis entre les mains de manipulateurs non spécialisés.

Il est à souhaiter que cette méthode trouve auprès des organismes de contrôle chargés de dépister l'addition de laits étrangers au lait humain tout l'appui qu'ils désirent, toute la commodité et la rapidité d'exécution qu'ils souhaitent.

\section{LA MÉTHODE DE CONTROLE ET DE CONSERVATION \\ DU LAIT MATERNEL AU LACTARIUM (1)}

par

A. ROSSIER

Médeoin des Hôpitaux de Paris

\section{JACQUELINE BERTRAND}

Directrice technique du Lactarium

Le Lactarium de l'Ecole de Puériculture compte aujourd'hui deux années d'existence (2)

Fondé en avril 1947 pour répondre à une demande pressante, il peut actuellement, grâce à une organisation de plus en plus poussée, assurer du lait maternel aux nourrissons débiles, prématurés et malades qui en ont un besoin vital.

Le principe même du lactarium, qui est de collecter le lait maternel à domicile, suppose et exige la mise en œuvre, d'une manière systématique, d'une série d'examen's de contrôle pratiqués chaque jour au laboratoire spécialisé du lactarium.

Nous voulons, dans cet article, exposer la méthode de contrôle et de conservation du lait maternel.

\section{CONTROLE DE LA PROPRETÉ}

\section{A. Acidimétrie}

Un test indirect, mais très sensible de la propreté du lait récolté est fourni par son taux d'acidité. Il renseigne, en effet, indirectement sur la mánière dont le lait a été recueilli. Une hygiène défectueuse de la traite, une conservation dans un récipient insuffisamment nettoyé ou à une température insuffisamment fraîche se traduira, en effet, par un excès d'acidité dû au développement de la flore lactiquel de fermentation. Il peut même arriver, dans les conditions les moins bonnes, que le lait “ tourne ». L'acidité est, d'autre part, plus élevée en été qu'en hiver.

Normalement, un bon lait maternel doit renfermer moins de

(1) L'Alimentation et la Vie, 1949, 37, 4-5-6-111.

(2) Voir Le Lait, 1948, 28, 333. 